\title{
Neuroendocrine differentiation of prostate cancer leads to PSMA suppression
}

\author{
Martin K Bakht1,2,3, Iulian Derecichei3, Yinan Li4, Rosa-Maria Ferraiuolo3, Mark Dunning5, So Won Oh', \\ Abdulkadir Hussein' ${ }^{6}$, Hyewon Youn ${ }^{1,2,7,8}$, Keith F Stringer 3,9, Chang Wook Jeong10, Gi Jeong Cheon ${ }^{1,2}$, Cheol Kwak ${ }^{10}$, \\ Keon Wook Kang1,2, Alastair D Lamb5,11, Yuzhuo Wang4,12, Xuesen Dong ${ }^{4}$ and Lisa A Porter ${ }^{3}$ \\ 'Department of Nuclear Medicine, Seoul National University College of Medicine, Seoul, Korea \\ 2Laboratory of Molecular Imaging and Therapy, Cancer Research Institute, Seoul National University College of Medicine, Seoul, Korea \\ 3Department of Biological Sciences, University of Windsor, Windsor, Ontario, Canada \\ ${ }^{4}$ Vancouver Prostate Centre, Department of Urologic Sciences, University of British Columbia, Vancouver, British Columbia, Canada \\ ${ }^{5}$ Cancer Research UK Cambridge Institute, University of Cambridge, Cambridge, UK \\ ${ }^{6}$ Department of Mathematics and Statistics, University of Windsor, Windsor, Ontario, Canada \\ 7Tumor Microenvironment Global Core Research Center, Seoul National University, Seoul, Korea \\ ${ }^{8}$ Cancer Imaging Center, Seoul National University Hospital, Seoul, Korea \\ 9Department of Pathology, Cincinnati Children's Hospital Medical Center, Cincinnati, Ohio, USA \\ ${ }^{10}$ Department of Urology, Seoul National University College of Medicine, Seoul, Korea \\ ${ }^{11}$ Nuffield Department of Surgical Sciences, University of Oxford, Oxford, UK \\ ${ }^{12}$ Department of Experimental Therapeutics, BC Cancer Research Centre, Vancouver, British Columbia, Canada
}

Correspondence should be addressed to G J Cheon or L A Porter: larrycheon@snu.ac.kr or Iporter@uwindsor.ca

\begin{abstract}
Prostate-specific membrane antigen (PSMA) is overexpressed in most prostate adenocarcinoma (AdPC) cells and acts as a target for molecular imaging. However, some case reports indicate that PSMA-targeted imaging could be ineffectual for delineation of neuroendocrine (NE) prostate cancer (NEPC) lesions due to the suppression of the PSMA gene (FOLH1). These same reports suggest that targeting somatostatin receptor type 2 (SSTR2) could be an alternative diagnostic target for NEPC patients. This study evaluates the correlation between expression of FOLH1, NEPC marker genes and SSTR2. We evaluated the transcript abundance for FOLH1 and SSTR2 genes as well as NE markers across 909 tumors. A significant suppression of FOLH1 in NEPC patient samples and AdPC samples with high expression of NE marker genes was observed. We also investigated protein alterations of PSMA and SSTR2 in an NE-induced cell line derived by hormone depletion and lineage plasticity by loss of p53. PSMA is suppressed following NE induction and cellular plasticity in p53-deficient NEPC model. The PSMA-suppressed cells have more colony formation ability and resistance to enzalutamide treatment. Conversely, SSTR2 was only elevated following hormone depletion. In 18 NEPC patient-derived xenograft (PDX) models we find a significant suppression of FOLH1 and amplification of SSTR2 expression. Due to the observed FOLH1-supressed signature of NEPC, this study cautions on the reliability of using PMSA as a target for molecular imaging of NEPC. The observed elevation of SSTR2 in NEPC supports the possible ability of SSTR2-targeted imaging for follow-up imaging of low PSMA patients and monitoring for NEPC development.
\end{abstract}

\section{Key Words}

- prostate cancer

- PSMA

- neuroendocrine differentiation

- nuclear medicine

- somatostatin receptor https://erc bioscientifica.com https://doi.org/10.1530/ERC-18-0226
C 2019 Society for Endocrinology Published by Bioscientifica Ltd. Printed in Great Britain
Endocrine-Related Cancer (2019) 26, 131-146 


\section{Introduction}

The main treatment protocol for patients suffering from castration-resistant prostate cancer (CRPC) is androgen receptor pathway inhibition (ARPI). Selection pressure and lineage plasticity of ARPI can lead to neuroendocrine (NE) differentiation of prostate adenocarcinoma (AdPC), promoting the more prevalent subtype of CRPC, which is termed treatment-induced neuroendocrine prostate cancer (NEPC) (Davies et al. 2018). It has been speculated that mutations in lineage regulators such as retinoblastoma $1(\mathrm{Rb} 1)$ and tumor protein 53 (p53) could also pave the way of ARPI to confer AdPC lineage plasticity and development of NEPC (Chen et al. 2018).

Due to the presumed androgen receptor (AR) negativity of NEPC tumors, treatment options are restricted to platinum- and cisplatin-based combinations and median survival of NEPC patients is much lower than patients with AdPC (Vlachostergios \& Papandreou 2015). Early identification of NEPC and novel targeting options could be beneficial. The transmembrane protein prostatespecific membrane antigen (PSMA) is an appealing target for molecular imaging and therapy of AdPC since it is overexpressed in a majority of AdPC tumors and metastatic lesions (Rai et al. 2016). Targeting PSMA is feasible by a wide variety of radioligands (Kopka et al. 2017, Rowe et al. 2017, Jadvar \& Ballas 2018). The PSMA ligands can also be labeled with therapeutic radionuclides which can irradiate PSMA-expressing cells (Boegemann et al. 2017). Despite the positive implications of PSMA for many forms of advanced AdPC there are clinical reports supporting that PSMA-targeted imaging is not able to delineate NEPC tumors (Parimi et al. 2014, Chakraborty et al. 2015, Sheikhbahaei et al. 2017, Tosoian et al. 2017, Usmani et al. 2017). In four specific cases, NEPC patients did not show substantial PSMA-radioligand uptake, in one case, this was described due to a downregulation of PSMA (Chakraborty et al. 2015, Tosoian et al. 2017, Usmani et al. 2017, Parida et al. 2018). To date, the relevance of these clinical reports has not been investigated.

NEPC tumors express common markers such as neuron-specific enolase (NSE; gene ENO2), chromogranin A (CHGA) and synaptophysin (SYP) (Parimi et al. 2014). NEPC is associated with the loss of RE1-silencing transcription factor (REST) due to alternate splicing by the RNA splicing factor serine/arginine repetitive matrix 4 (SRRM4). We revealed that SRRM4 plays a key role in transdifferentiation of AdPC to NEPC under ARPI (Li et al. 2017). The somatostatin receptor subtype 2 (SSTR-2) is prevalent in the majority NE tumors and
DOTATOC and DOTATATE radioligands with affinity to SSTR are radiotracers for delineation of NE tumors (Poeppel et al. 2011). Recently, Gofrit et al. suggested that ${ }^{68} \mathrm{Ga}$-DOTATATE might be a potential option for NEPC imaging (Gofrit et al. 2017). Interestingly, Usmani et al. compared ${ }^{68} \mathrm{Ga}$-PSMA vs ${ }^{68} \mathrm{Ga}$-DOTA in a NEPC patient and reported that the NEPC tumor and metastatic lesions have avid uptake of ${ }^{68} \mathrm{Ga}$-DOTA while the ${ }^{68} \mathrm{Ga}$-PSMA uptake was faint and inconclusive (Usmani et al. 2017).

In this study, we use bioinformatic datasets, cell lines and patient-derived xenograft (PDX) models to study the correlation between expression of the PSMA gene, FOLH1, NE biomarkers and SSTR2. This work supports that NEPC tumors have a distinct PSMA-suppressed signature and demonstrate the possibility that SSTR2-targeted imaging could be an alternative diagnostic target for this aggressive form of prostate cancer.

\section{Materials and methods}

\section{Cell lines and cell culture}

The LNCaP and DU-145 cell lines were purchased from ATCC. The high passage LNCaP cell line (LNCaP-HP) was a generous gift from Dr Dora Cavallo-Medved of University of Windsor. LNCaP and LNCaP-HP cells were grown in RPMI-1640 in the presence of 10\% fetal bovine serum (FBS). DU-145 cells were grown in Eagle's minimum essential medium (E-MEM) supplemented with 10\% FBS. For induction of NE transdifferentiation, LNCaP cells were cultured in RPMI-1640 medium with 10\% charcoalstripped serum (CSS) over ten passages during 4 weeks. LNCaP cells were cultured in RPMI medium supplemented with $10 \%$ CSS when treated with $10 \mu \mathrm{M}$ enzalutamide (ENZ) from Selleckchem (Houston, TX, USA).

\section{Plasmids and infection}

Small hairpin RNA (shRNA)-based p53 knockdown was attained by transducing LNCaP cells with p53 shRNA lentiviral particles and transduction control cells were produced by using control shRNA lentiviral particles. pLKO1 shp53-targeting shRNA (Addgene, \#19119) $(\mathrm{MOI}=8)$ and pLKO1-control (Addgene, \#8453) as an empty backbone were gifts from Dr Bob Weinberg of Massachusetts Institute of Technology. The lentiviral production was previously described (Al Sorkhy et al. 2012). Overall, 10,000 cells were seeded in fully supplemented growth media in 24 -well plates for $24 \mathrm{~h}$. Cells were starved by removing serum from the media, followed by the use https://erc bioscientifica.com https://doi.org/10.1530/ERC-18-0226
C) 2019 Society for Endocrinology Published by Bioscientifica Ltd. Printed in Great Britain 
of $1 \mathrm{mg} / \mathrm{mL}$ polybrene (Santa Cruz, sc-134220) and MOI 3 of the specific vector used. Infected media was changed to fully supplemented media $24 \mathrm{~h}$ after infection. Cells were incubated with $1 \mathrm{mg} / \mathrm{mL}$ puromycin (Sigma, P8833) $48 \mathrm{~h}$ after infection for $72 \mathrm{~h}$ to allow for puromycin selection. Media is thereafter changed every $48 \mathrm{~h}$ with puromycin included. The isolated single-cell clones were cultured in RPMI medium supplemented with 10\% CSS.

\section{Immunoblotting and immunocytochemistry}

Immunoblotting and immunocytochemistry techniques were described previously (da Silva et al. 2016, Li et al. 2017). In brief, cells were lysed in TNE buffer $(50 \mathrm{mM}$ Tris, $150 \mathrm{mM} \mathrm{NaCl}, 5 \mathrm{mM}$ EDTA) with protease inhibitors (leupeptin $2 \mu \mathrm{g} / \mathrm{mL}$, aprotinin $5 \mu \mathrm{g} / \mathrm{mL}$, PMSF $100 \mu \mathrm{g} / \mathrm{mL}$ ). Protein concentrations were assessed using the Bradford assay and equal amounts of protein were analyzed using SDS-PAGE and transferred to PVDF membranes. Membranes were blocked for $1 \mathrm{~h}$ at room temperature in $1 \%$ BSA and incubated in primary antibody overnight at $4^{\circ} \mathrm{C}$, followed by secondary at a concentration of $1: 10,000$ for $1 \mathrm{~h}$ at room temperature. Visualization was conducted using chemiluminescent peroxidase substrate (Pierce) as per manufacturer's instructions. Images were captured on Alpha Innotech HD 2 using AlphaEase FC software.

For immunocytochemistry, primary antibodies were diluted in 3\% BSA-0.1\% Tween-20 in 1× PBS and used at a concentration of $1: 200$. Secondary antibodies were used at a concentration of 1:750. Slides were imaged using the LEICA DMI6000 inverted microscope with LAS 3.6 software.

Actin antibody was purchased from ChemiconMillipore (MAB150 1R). PSMA (D4S1F) was purchased from Cell Signaling (12702). The secondary rabbit and mouse antibodies were purchased from Sigma. SSTR2 (sc-365502), AR (sc-518), NSE (sc-271384) and p53 (sc-53394) were obtained from Santa Cruz Biotechnology.

\section{Cell proliferation assay}

Cell proliferation assay was conducted by plating 20,000 LNCaP cells per well of a 24 -well cell culture plate in $500 \mu \mathrm{L}$ of RPMI medium supplemented with 10\% CSS and treated with vehicle control (DMSO) or enzalutamide $(10 \mu \mathrm{M})$ for 6 days. Cell numbers were counted using trypan blue exclusion and using a hemocytometer; counts were also verified using a TC10 automated cell counter (Biorad).

\section{Colony formation assay}

To evaluate the anchorage-independent ability of LNCaP cells with different levels of PSMA, the colony formation assay was used. LNCaP cells were seeded in six-well plates at a concentration of 2500 cells per well. They were then cultured for 1 week in RPMI supplemented with CSS, followed by treatment for one more week with vehicle control (DMSO) or enzalutamide $(10 \mu \mathrm{M})$. The cell colonies were fixed with $4 \%$ paraformaldehyde and stained with crystal violet (Sigma-Aldrich). Colonies were photographed and scored via NIH ImageJ software.

\section{Neurite length measurement and statistical analysis}

Neurite length for each separate cell was measured by manual tracing and determined using NIH ImageJ software as previously described (Ding et al. 2015). The neurites were defined as a process with lengths equivalent to one diameters of a cell body. The percentage of neuritebearing cells was calculated from the total number of counted cells ( $n=3, \sim 1000$ cells measured).

\section{Quantitative real-time PCR analysis}

RNA was isolated using Qiagen RNeasy Plus Mini Kit as per manufacturer's instructions. cDNA was synthesized using Superscript II (Invitrogen) as per manufacturer's instructions. SYBR Green detection (Applied Biosystems) was used for real-time PCR and was performed and analyzed using Viia7 Real-Time PCR System (Life Technologies) and software. The primers used are listed on Supplementary Table 1 (see section on supplementary data given at the end of this article).

\section{In silico dataset}

Using Human Protein Atlas (www.proteinatlas.org) (Thul et al. 2017, Uhlen et al. 2017), cBioPortal web-portal (http://cbioportal.org) (Gao et al. 2013) and the web-portal UALCAN (Chandrashekar et al. 2017), we evaluated the transcript abundance for PSMA gene (FOLH1), prostatespecific antigen (PSA) gene (KLK3), four well-known NE biomarker genes including NSE gene (ENO2), CD56 gene (NCAM1), synaptophysin gene (SYP), chromogranin A gene (CHGA).

Cambridge Carcinoma of the Prostate App (http:// bioinformatics.cruk.cam.ac.uk/apps/camcAPP) as a (c) 2019 Society for Endocrinology Published by Bioscientifica Ltd. Printed in Great Britain 
Table 1 An overview of the prostate cancer datasets used in this study.

\begin{tabular}{|c|c|}
\hline Dataset name & Source \\
\hline Cambridge 2015 & Ross-Adams et al. (2015) \\
\hline MSKCC 2010 & Taylor et al. (2010) \\
\hline Michigan 2012 & Grasso et al. (2012) \\
\hline $\begin{array}{l}\text { TCGA for prostate } \\
\text { cancer }\end{array}$ & UALCAN (Chandrashekar et al. 2017) \\
\hline Beltran 2016 & Beltran et al. (2016) \\
\hline
\end{tabular}

multiple AdPC genomic datasets was used in this study (Dunning et al. 2017). The web-portal UALCAN (Chandrashekar et al. 2017) was also used which focuses on TCGA level 3 RNA-seq and clinical data from 31 cancer types. We analyzed relative expression of genes across AdPC tumors and normal samples, based on Gleason grading system using this resource. In addition, we used the Beltran dataset (Beltran et al. 2016) including 34 AdPC samples from 33 patients and 15 NEPC samples from 10 patients. Table 1 provides an overview of the datasets used in analysis.

The expression of PSMA protein was examined in a variety of organs using the Human Protein Atlas (www. proteinatlas.org). Two anti-PSMA antibodies including HPA010593 (Sigma-Aldrich) and CAB001451 (Novocastra) were used for visualization and measurement of PSMA in all major tissues and organs $(n=45)$ in the human body, supplemented with RNA-sequencing data for 31 tissues. The same antibodies used for immunohistochemistry images of AdPC tissues in different stages and each annotated by pathologists (Thul et al. 2017, Uhlen et al. 2017).

\section{The survival data and pairwise-correlations of gene expression}

The specialized web interface, Cambridge Carcinoma of the Prostate App (http://bioinformatics.cruk.cam.ac.uk/ apps/camcAPP/) as a source for multiple AdPC genomic datasets was used in this study (Dunning et al. 2017). Table 1 provides an overview of the datasets used in this analysis (Taylor et al. 2010, Ross-Adams et al. 2015). The Cambridge (Ross-Adams et al. 2015) cohort involved 358 fresh frozen samples from 156 patients comprising; 125 primary prostate cancer from radical prostatectomy with matched benign tissue, 64 matched germline genomic DNA (gDNA), 19 CRPC from channel transurethral

\begin{tabular}{l}
\hline Platform: gene expression \\
Illumina HT12 \\
Affymetrix Human $1.0 \mathrm{ST}$ \\
Agilent Whole Human 44k \\
Agilent 2100
\end{tabular}

Platform: copy number
Illumina Omni 2.5
Agilent $244 \mathrm{k}$
Agilent $105 \mathrm{k} / 244 \mathrm{k}$
Illumina HiSeq $2000 \mathrm{~N}$
Illumina HiSeq

Sample size and

tumor type

125 Primary

19 Advanced

109 Primary

19 Advanced

59 Primary

32 Advanced

497 Primary

34 AdPC 15 NEPC resection of the prostate, 13 with matched germline gDNA, and 12 independent benign samples from holmium laser enucleation of the prostate. The camcAPP was used for recursive partitioning-based survival analysis and KaplanMeier plots, pairwise-correlations of gene expression and heatmaps of gene expression data.

Survival data and heatmaps were generated using camcAPP, which is implemented in $\mathrm{R}$ code as a Shiny application (Dunning et al. 2017). Kaplan-Meier biochemical relapse-free survival plots were generated using a recursive partitioning analysis named unbiased recursive partitioning (Hothorn et al. 2006). This conditional inference framework was used to determine if the samples could be split into groups based on the expression data from each of the genes of interest. The algorithm tailored for our case can be described as follows: a statistical test of independence was run between gene expression levels and the survival times. When the $P$ value of initial test of independence (ITI) was found to be less than 0.05 , an optimal cutoff point was determined in the expression data such that a weighted log-rank statistic (a loss function based on log-rank) comparing the two groups resulting from dividing the sample of patients by the cutoff point would be maximized. Afterward, the samples were split at the optimal cutoff point on the expression scale and represented as a log-rank comparing those two groups. When the algorithm did not confirm dependence between survival times and gene expression, we split samples into two groups based on median expression level of the gene. In the cases with ITI $P$ values more than 0.05 , we are unable to state a definitive relationship between the expression of the gene and survival. The value quoted on the Kaplan-Meier plots of this manuscript indicate where the recursive partitioning algorithm has found two distinct groups of samples as high and low expression levels and the log-rank test was employed to identify statistical difference between https://erc bioscientifica com

https://doi.org/10.1530/ERC-18-0226
C) 2019 Society for Endocrinology Published by Bioscientifica Ltd. Printed in Great Britain 
the high and low expressing groups. Pearson correlation was used for pairwise-correlations of the studied gene expression analysis.

\section{Animals and PDX models}

Fresh AdPC or NEPC tissues from patients were grafted under the kidney capsules of non-obese diabetic/severe combined immunodeficient (NOD/SCID) mice. This study followed the ethical guidelines stated in the Declaration of Helsinki, specimens were obtained from patients with their informed written consent approved by the Institutional Review Board of the University of British Columbia (UBC). Animal care and experimental procedures were carried out in accordance with the guidelines of the Canadian Council on Animal Care (CCAC) under the approval of the Animal Care Committee of the UBC.

The expression of the PSMA gene in five AdPC PDX models (LTL-313-B, LTL-313-B-R, LTL-418, LTL-418-R and LTL-331-7) and two NEPC PDX models (LTL-331-7-R and LTL-352) was studied by real-time PCR analysis. Transcription of FOLH1 and SSTR2 genes in 18 PDX models including three NEPC models were analyzed. Transcriptomic analysis for all PDX models, with the exception of the LTL331-331R castration time-series samples, was achieved by GE $8 \times 60 \mathrm{~K}$ microarray and transcriptomic analysis of the LTL331-331R time-series performed using RNA-sequencing data (Akamatsu et al. 2015, Ci et al. 2018). We previously characterized and validated these models as having AdPC and NEPC mRNA and protein signatures (Li et al. 2017, Lin et al. 2014, Nabavi et al. 2017).

\section{Statistical analysis}

All of the in vitro experiments were performed in triplicates and repeated three times. All in vitro and in vivo results are expressed as the mean \pm standard error of the mean (s.E.M.). In the box whisker plots, the line inside each box is the median, upper box border represents the 75th quartile, lower box border represents the 25 th quartile and whiskers represent the range. Statistical analysis was done using the GraphPad Prism 5.01 software (GraphPad Software). Differences between the two groups were compared by unpaired Student's $t$-test. One-way ANOVA followed by a Benjamini-Hochberg, Tukey or Newman-Keuls multiple comparison test was used to compare differences among multiple groups. The false discovery rate (FDR) less than 0.05 considered as significant in Benjamini-Hochberg adjustment. The levels of significance were set at $P<0.05$ as *, $P<0.01$ as ** and $P<0.001$ as ***.

For quantification of FOLH1 heatmaps, $-0.5<Z$-score and $+0.5>Z$-score are considered as suppression and amplification thresholds. The percent of patients with suppression ( $Z$-score $\leq-0.5)$, no alteration $(-0.5<Z$-score $<+0.5)$ and amplification ( $Z$-score $\geq+0.5)$ of FOLH1 in each group of samples were calculated. Total number of patients with amplification of the gene of interests were counted and set as the maximum value. Of the maximum value patients, those who were co-upregulated with FOLH1 were categorized as 'Amplification', while those whose FOLH1 status did not change or was downregulated were categorized as 'No Alteration' and 'Suppressed' respectively. The values for all three categories were then divided by the maximum value and graphed under their respective categories.

\section{Results}

\section{High-grade CRPC has inconsistent expression of the PSMA gene (FOLH1)}

FOLH1 expression was highly upregulated in prostate tissue and relatively upregulated in both the hippocampus and salivary gland (Supplementary Fig. 1A). Similarly, protein level of PSMA was higher in prostate tissue than all other organs and tissues. Only the kidney, small intestine and duodenum reached medium levels of protein expression (Supplementary Fig. 1B). As compared to a spectrum of other cancer types (Gao et al. 2013), FOLH1 is highly amplified in AdPC (Supplementary Fig. 2A). AdPC patients with amplification in FOLH1 have a significant poorer survival rate in MSKCC (Taylor et al. 2010) dataset (Supplementary Fig. 2B). Similarly, the Cambridge (RossAdams et al. 2015) dataset displayed differential outcomes for men with low vs high FOLH1 expression (log-rank $P=0.047)$; however, the initial test of independence shows no significance and hence conclusions cannot be drawn from this dataset (ITI $P=0.31$ ).

With the aim of exploring PSMA gene levels during progression of AdPC to clinically relevant CRPC, the Michigan and Cambridge datasets were used and gene expression levels studied (Fig. 1A and B). The Michigan dataset (Grasso et al. 2012) showed that there is an expected rise from normal to AdPC tissue in expression of FOLH1, but there was no statistically significant difference between hormone-responsive AdPC and CRPC tumors (Fig. 1A). A cluster of samples with very low expression of the PSMA gene is observable in CRPC https://erc.bioscientifica.com

https://doi.org/10.1530/ERC-18-0226 (c) 2019 Society for Endocrinology Published by Bioscientifica Ltd. Printed in Great Britain 

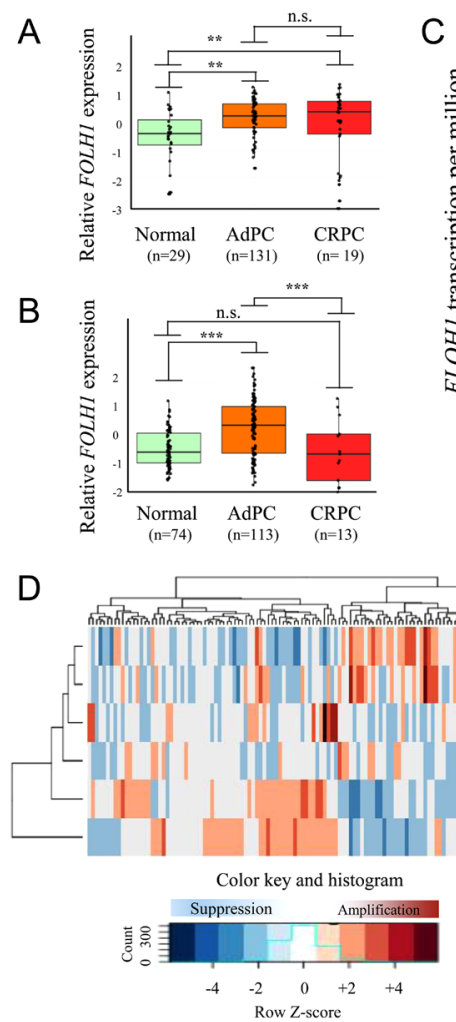

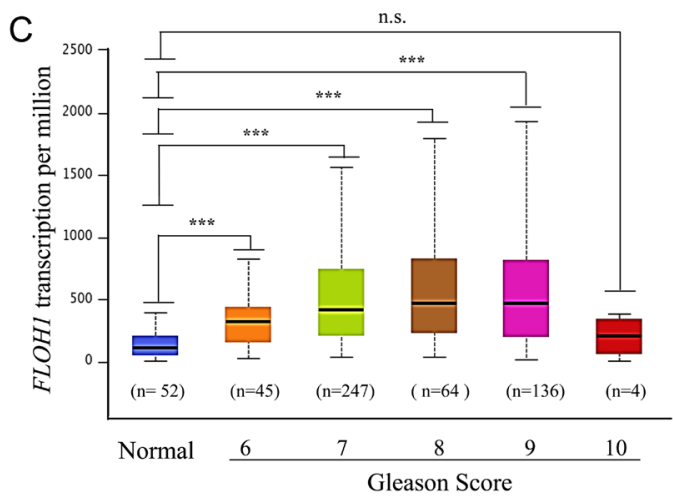

E

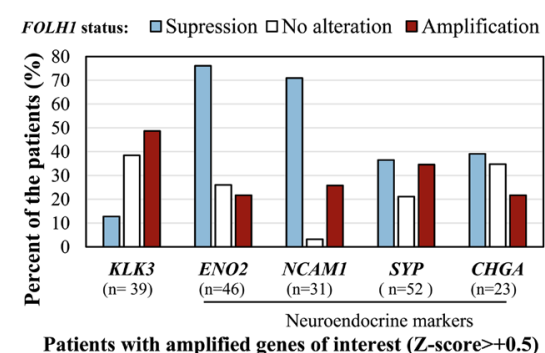

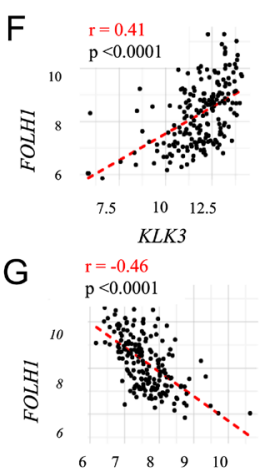

ENO2

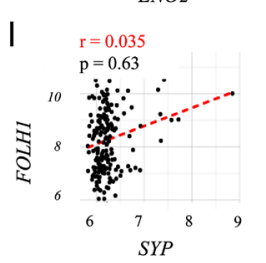

SYP
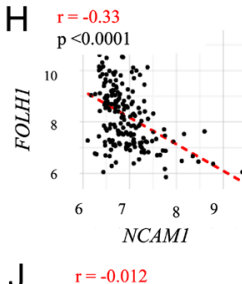

J $\quad r=-0.012$

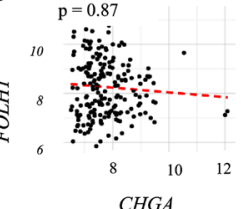

Figure 1

Expression of PSMA at varying grades of CRPC. (A and B) Box-whisker plots showing the expression of FOLH1 gene in three different classes of samples from (A) Michigan (Grasso et al. 2012) and (B) Cambridge (Ross-Adams et al. 2015) datasets. (C) The expression of FOLH1 during progression of AdPC based on Gleason score from TCGA dataset generated by web-portal UALCAN (Chandrashekar et al. 2017). One-way ANOVA followed by unpaired t-tests were performed with Benjamini-Hochberg adjustment for multiple test correction; $* \star p<0.01$ and $* \star \star p<0.001$, n.s.: no significant. (D) Heatmap plot of the mean expression levels of FOLH1, PSA gene (KLK3) and four major clinically significant NE marker genes including among patients of Cambridge (Ross-Adams et al. 2015) datasets. (E) Percent of patients with suppression (Z-score $\leq+0.5$ ), no alteration ( $-0.5<Z$-score $<+0.5)$ and amplification (Z-score $\geq+0.5$ ) of FOLH1 in each group of samples. (F, G, H, I and J) Pairwise correlations of the studied gene expression and Pearson correlation analysis from Cambridge (Ross-Adams et al. 2015) datasets. A full-colour version of this figure is available at https://doi.org/10.1530/ERC-18-0226.

samples. Similarly, Fig. 1B illustrates an increase from benign to hormone-responsive tumor in the expression of FOLH1 in the Cambridge dataset and, interestingly, there was a significant drop in expression when transitioning to CRPC. To further explore this inconsistency, we evaluated the expression of FOLH1 by Gleason grading score (Fig. 1C) in TCGA dataset (Chandrashekar et al. 2017). From benign tissue (normal) to Gleason score (GS) 8 FOLH1 levels steadily increase and then they remain unchanged between the transition from 8 to 9 . Samples with GS of ten showed no significant elevation in comparison with the normal samples. While the sample size $(n=4)$ prevents definitive conclusions for GS 10, the observed fall in FOLH1 expression supports the possibility of a suppression in high-grad AdPC. Collectively, this led us to conclude that expression of FOLH1 in high-grade CRPC is variable and that there is a need to question whether FOLH1 expression and PSMA protein levels are effective in determining progression to the highest grades of AdPC.

\section{An inverse correlation between the expression of FOLH1 and common NE biomarker genes}

Figure 1D shows the mean expression levels of FOLH1, PSA gene (KLK3) and NE marker genes over 144 patients. As we expected, KLK3 and FOLH1 expression had a direct correlation where $49 \%$ of patients with amplified $K L K 3$ also showed an amplification of FOLH1 (Fig. 1E). In contrast, an inverse correlation between FOLH1 and NE biomarker gene expression was identified. Particularly, suppression of FOLH1 was observed in 65 and 53\% of patients who were overexpressing the NE genes ENO2 and NCAM1, respectively. The numbers of patients with amplification of genes of interest based on level of FOLH1 gene expression are presented on Supplementary Table 2. In Fig. 1F, G, H, I and J,
C) 2019 Society for Endocrinology Published by Bioscientifica Ltd. Printed in Great Britain 
this relationship was further characterized through the use of a Pearson correlation. On Fig. $1 \mathrm{G}$ and H, a strong inverse correlation between FOLH1 and ENO2 and NCAM1 is prevalent ( $r=-0.46$ and -0.33 ), while correlation between FOLH1 and the other NE markers was not significant. Figure $1 \mathrm{~F}$ shows there was a significant direct correlation between FOLH1 and KLK3 genes $(r=0.41)$. Supplementary Table 3 provides confidence interval (CI) parameters of these gene correlations. Supplementary Figure 3 shows the mean expression levels of FOLH1, NE genes, AR and AR target genes. Similar to the PSA gene (KLK3), $A R$ and some other $A R$ target genes such as KLK2, FKBP5, CAMKK2 and NKX3-1 are also inversely correlated with FOLH1 expression. The overall regression analysis led us to conclude that PSMA and NE biomarkers are inversely correlated.

\section{Treatment-induced NEPC correlates with PSMA suppression}

Previously we showed SRRM4 can induce NEPC in patients treated by ARPI through compromising the function of genes such as REST (Li et al. 2017). The elevation of SRRM4 and the loss of REST are indicators of treatmentinduced NEPC. Figure 2A and B show that when SRRM4 is amplified FOLH1 is highly suppressed and, conversely, when REST is amplified FOLH1 is amplified. Specifically, the suppression of FOLH1 was observed in $57 \%$ of patients who were overexpressing SRRM4, while only $15 \%$ of patients who were overexpressing REST had suppression of FOLH1.

As summarized on Table 1, Beltran dataset (Beltran et al . 2016) patient samples are histologically characterized as AdPC and NEPC. Figure $2 \mathrm{C}$ shows NEPC has significantly lower expression of FOLH1 $(P<0.001)$ as compared to AdPC. Following this we analyzed the association between FOLH1 and SRRM4 expression in NEPC samples and found that a significant $(P=0.011)$ inverse correlation was present between the two biomarkers $(r=-0.358)$ (Fig. 2D). Also, Fig. 2E shows FOLH1 directly correlated to REST expression $(r=0.561)$. The relationship of SRRM 4 and REST to FOLH1 collectively supports that treatment-induced NE transdifferentiation correlates with suppressed PSMA gene expression.

\section{An inverse correlation between the expression of FOLH1 and SSTR2}

The heatmap plot in Fig. 3A displays NE marker genes clustered together at the top which showed less difference

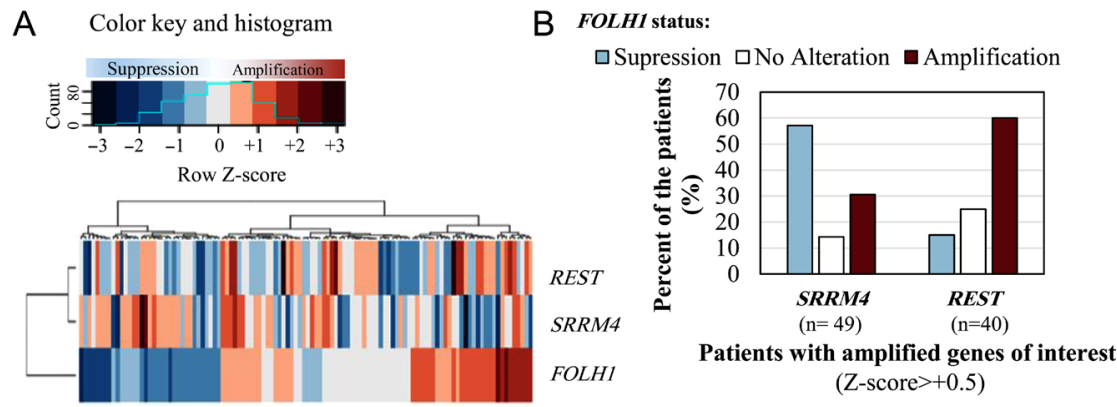

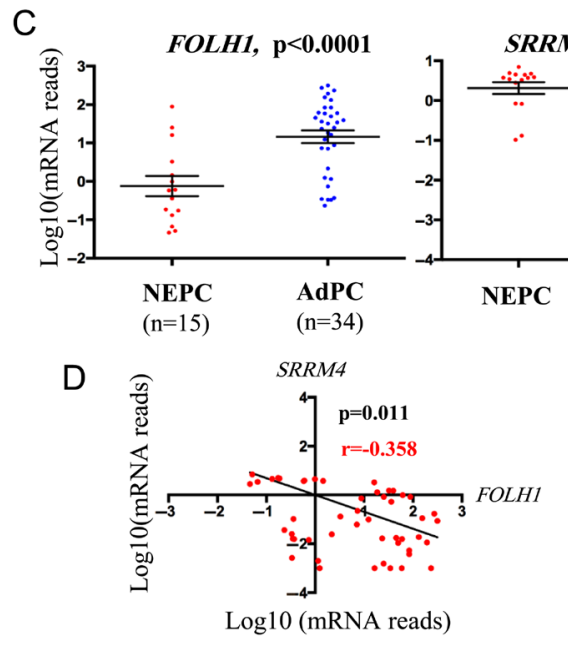

https://erc.bioscientifica.com https://doi.org/10.1530/ERC-18-0226 (c) 2019 Society for Endocrinology Published by Bioscientifica Ltd. Printed in Great Britain

\section{Figure 2}

Analysis of FOLH1, SRRM4 and REST in tumor datasets. (A) The heatmap plot of the mean expression levels of FOLH1, SRRM4 and REST genes among patients of Cambridge dataset (RossAdams et al. 2015). (B) The percent of patients with suppression (Z-score $\leq+0.5)$, no alteration $(-0.5<Z$-score $<+0.5)$ and amplification (Z-score $\geq+0.5$ ) of FOLH1 in each group of samples. (C) The comparison of FOLH1, SRRM4 and REST expressions between AdPC and NEPC samples of Beltran dataset (Beltran et al. 2016) Error bars reflect S.E.M. and Student's $t$-test was performed. (D, E) The relationship between FOLH1 and SRRM4 levels in NEPC samples in Beltran dataset (Beltran et al. 2016) by Pearson correlation analysis. A full-colour version of this figure is available at https://doi.org/10.1530/ ERC-18-0226. 
A

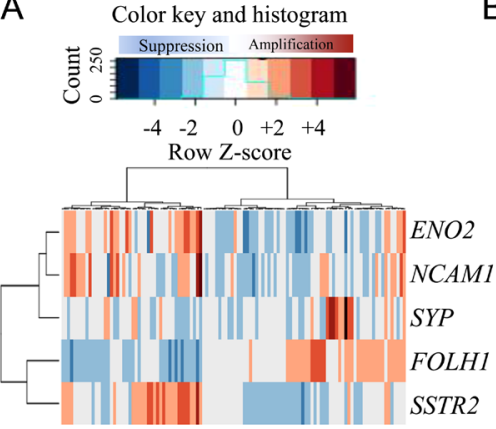

E

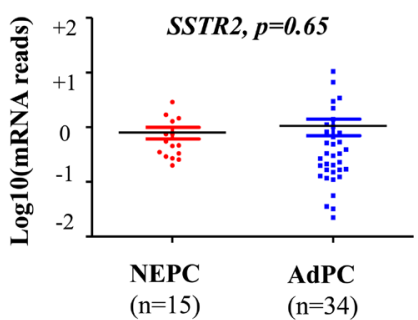

B FOLH1 status:

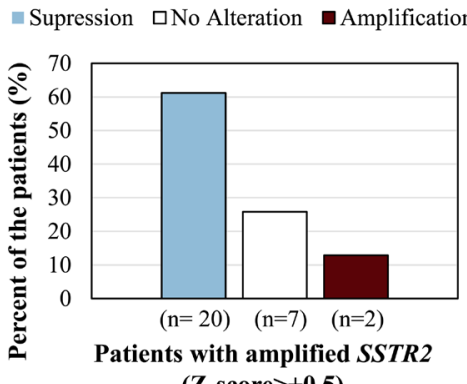

(Z-score $>+0.5$ )
C

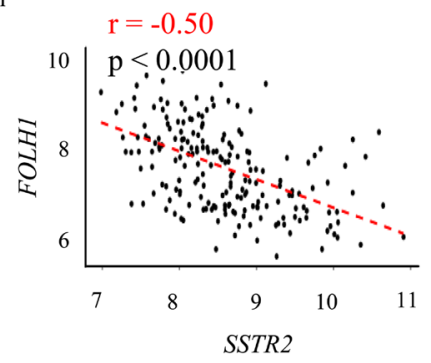

$\mathrm{D}$

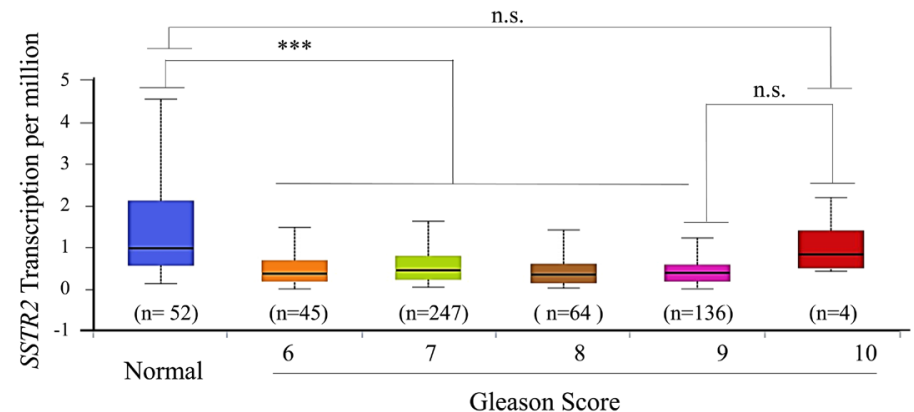

Figure 3

Correlative analysis of FOLH1 with SSRT2 and NE genes. (A) The heatmap plot of the mean expression levels of FOLH1, NE genes and somatostatin receptor-2 gene (SSTR2) expression among patients of Cambridge dataset (Ross-Adams et al. 2015) (method to calculate distances is euclidean). (B) The percent of patients with suppression ( $Z$-score $\leq+0.5$ ), no alteration $(-0.5<Z$-score $<+0.5)$ and amplification (Z-score $\geq+0.5)$ of $F O L H 1$ in each group of samples. (C) Pairwise correlation of treatment-induced gene expressions and Pearson correlation analysis from Cambridge dataset (Ross-Adams et al. 2015). (D) The expression of SSTR2 during progression of AdPC based on Gleason score from TCGA dataset generated by web-portal UALCAN (Chandrashekar et al. 2017). One-way ANOVA followed by a $t$-test was performed with Benjamini-Hochberg adjustment for multiple test correction; $\star \star P<0.01$ and $* * * P<0.001$, n.s.: no significant. (E) The comparison of SSTR2 expressions between AdPC and NEPC samples of Beltran dataset (Beltran et al. 2016) Error bars reflect S.E.M. and Student's $t$-test was performed. A full-colour version of this figure is available at https://doi.org/10.1530/ ERC-18-0226.

while SSTR2 and FOLH1 represented the largest difference; consequently, Fig. 3B demonstrates that more than $61 \%$ of patients with amplified SSTR2 are FOLH1 suppressed. Figure 3C and Supplementary Table 3 show a modest inverse correlation between SSRT2 and FOLH1 $(r=-0.50)$. Figure 3D shows that from benign tissue (normal) to GS of 9, FOLH1 is suppressed while there is an evidence of a two-fold increase in the expression of SSTR2 at GS of $10(P<0.05)$. SSRT2 level in NEPC sample showed no suppression (Fig. 3E). This led us to conclude that the observed suppression of FOLH1 could be accompanied by SSTR2 gene overexpression at high-grade CRPC.

\section{NEPC-like patients have significantly worse survival rates than non-NEPC-like patients}

Kaplan-Meier survival curves studying high and low expression levels of the PSA gene, $K L K 3$, fail to reveal any consistent correlation with patient survival over two different datasets (Fig. 4A and Supplementary Fig. 4A). SSTR2 and NE genes including ENO2 and NCAM1 did not correlate with a difference in BCR (Fig. 4B, D, $\mathrm{H}$ and Supplementary Fig. 4B, D, H). Figure 4E and Supplementary Fig. 4E show that high expression of SYP was associated with decreased relapse-free survival (poor outcome) in both of studied datasets (log-rank $P<0.05)$. High expression of $C H G A$ was also correlated with poor outcome in MSKCC (Taylor et al. 2010) dataset (Fig. 4C). Similarly, Kaplan-Meier analysis of CHGA in Cambridge (Ross-Adams et al. 2015) dataset showed clear partitioning for men with low vs high CHGA expression (log-rank $P=0.025$ ); however, the initial test of independence was not significant and hence no conclusion can be drawn from this dataset (ITI $P=0.28$ ).

The evaluation of treatment-induced NEPC, SRRM4, showed the SRRM4 high group had a significantly (logrank $P=0.046)$ poorer prognosis than SRRM4 low group (Fig. 4F). This trend continued with its downstream target, REST; where REST high group had a significantly (log-rank $P=0.0056$ ) better prognosis than REST low group (Fig. 4G).

The relationships observed in MSKCC (Taylor et al. 2010) dataset were not consistently observed in the 
A

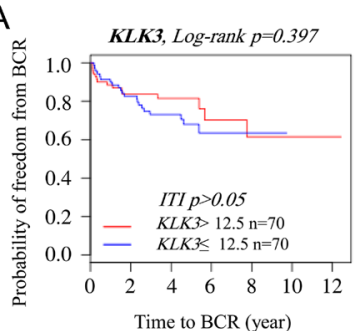

E

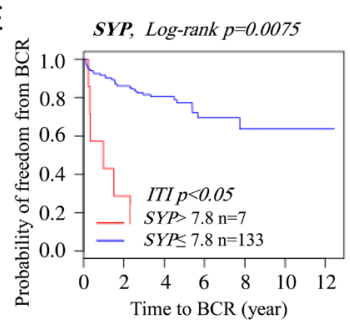

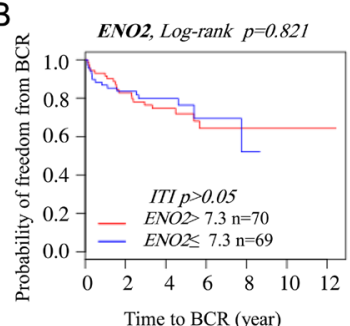

$\mathrm{F}$

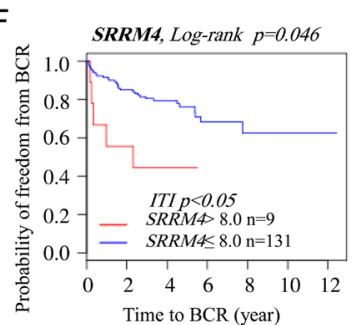

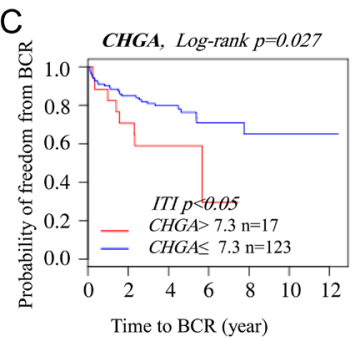

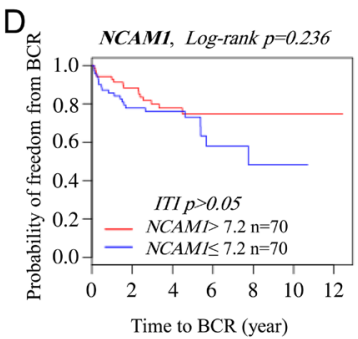

G

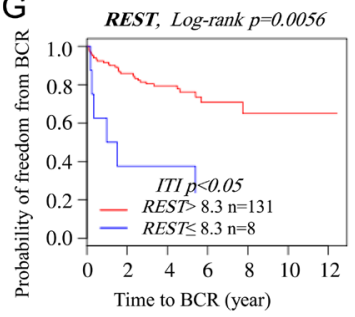

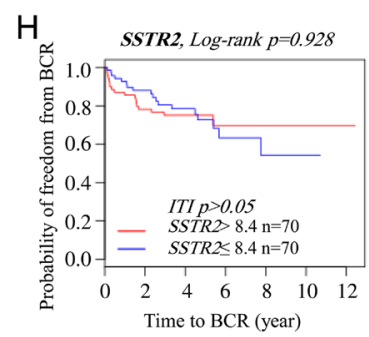

\section{Figure 4}

The probability of freedom from biochemical recurrence (BCR) of prostate cancer patients grouped according to the gene expression levels. Kaplan Meyer survival curves for high and low expression levels of (A) KLK3, (B) ENO2, (C) CHGA, (D) NCAM1, (E) SYP, (F) SRRM4, (G) REST, (H) SSTR2 genes generated by MSKCC (Taylor et al. 2010). A full-colour version of this figure is available at https://doi.org/10.1530/ERC-18-0226.

Cambridge (Ross-Adams et al. 2015) dataset. Results found non-significant in the Cambridge dataset do not prove that there is no relationship with survival for those genes, but this inconsistency provides evidence that the effects of several genes on survival are not clear-cut.

In all, the trends in Kaplan-Meier survival curves in Fig. 4 and Supplementary Fig. 2B, C imply that NE markers, especially treatment-induced NEPC, could be clinically significant in determining survival rating and correlate with poorer prognosis. Further examination into FOLH1 and $K L K 3$ is required before a definitive correlation can be made for these genes.

\section{PSMA suppression and SSTR2 overexpression in NE-induced AdPC cell line}

The levels of PSMA, SSTR2, AR and NSE were measured in three different cell line models representing androgensensitive AdPC, CRPC and NEPC (Fig. 5A and B). LNCaP cell line as an AR-positive cell line has wild-type p53, and it is considered a typical model for androgen-sensitive AdPC (Chen et al. 2018). In addition, LNCaP-HP as a high passage LNCaP cell line could be a model of CRPC due to its androgen insensitivity while it has positivity for AR (Unni et al. 2004). AR-negative DU145 cells, suggested to be used as a NEPC model, contain two different point mutations in the TP53 gene (Phe223Leu and Val274Phe), one on either allele, producing nonfunctional protein product (Chappell et al. 2012, Li et al. 2016). Figure 5B shows the level of NSE as a NE marker has continuous elevation from AdPC to NEPC. AdPC and CRPC in vitro models are AR and PSMA positive while NEPC models are PSMA and AR negative. SSTR2 has significantly higher level in DU145 model.

To examine the impact of NE transdifferentiation of AdPC on PSMA levels we used the LNCaP cell line which mimics the phenotype of NEPC cells when maintained overtime in steroid-reduced conditions (Zelivianski et al. 2001). LNCaP cells maintained in 10\% CSS for 1 month have an altered phenotype compared to control cells maintained in 10\% FBS (Fig. 5C). Almost 75\% of the CSS-treated LNCaP cells (LNCaP-CSS) extended neurites, whereas less than $10 \%$ of control or LNCaP-FBS cells bore neurites (Fig. 5D). Additionally, both the mean length of the longest neurite and the total neurite length were significantly increased in LNCaP-CSS cells compared with the LNCaP-FBS (Fig. 5E and F). These observations indicate that LNCaP growth conditions can play the role of in vitro models for AdPC and NEPC. The western blot assay (Fig. 5G and $\mathrm{H}$ ) demonstrated that SSTR2 and NSE as a NE marker protein are expressed at a higher level in the LNCaP-CSS cell line. In contrast, PSMA, AR and p53 proteins have a significant decline. Immunofluorescence microscopy of LNCaP cell lines supports western blot data (Fig. 5I, J, K and L). Collectively, these data demonstrate that LNCaP-CSS are differentiating toward a NE phenotype and that this occurs coincidentally with a decrease in PSMA and AR levels. (c) 2019 Society for Endocrinology Published by Bioscientifica Ltd. Printed in Great Britain 
A

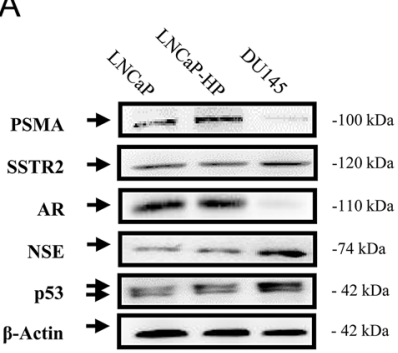

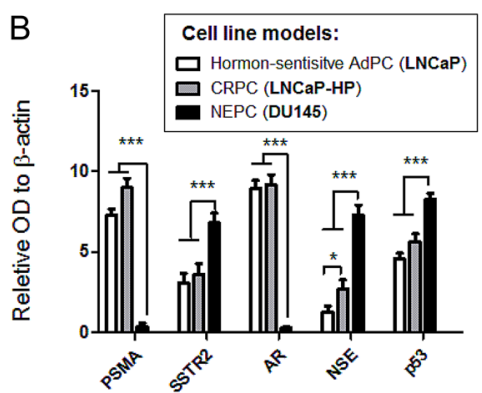

F

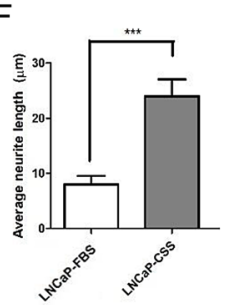

C

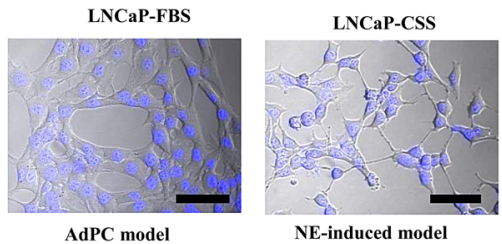

$\mathrm{H}$

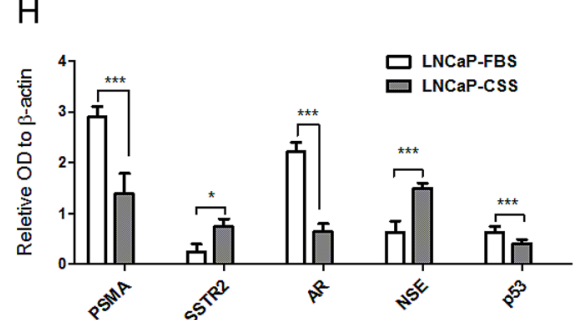

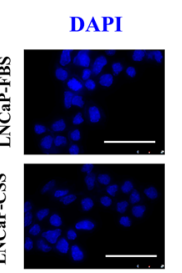
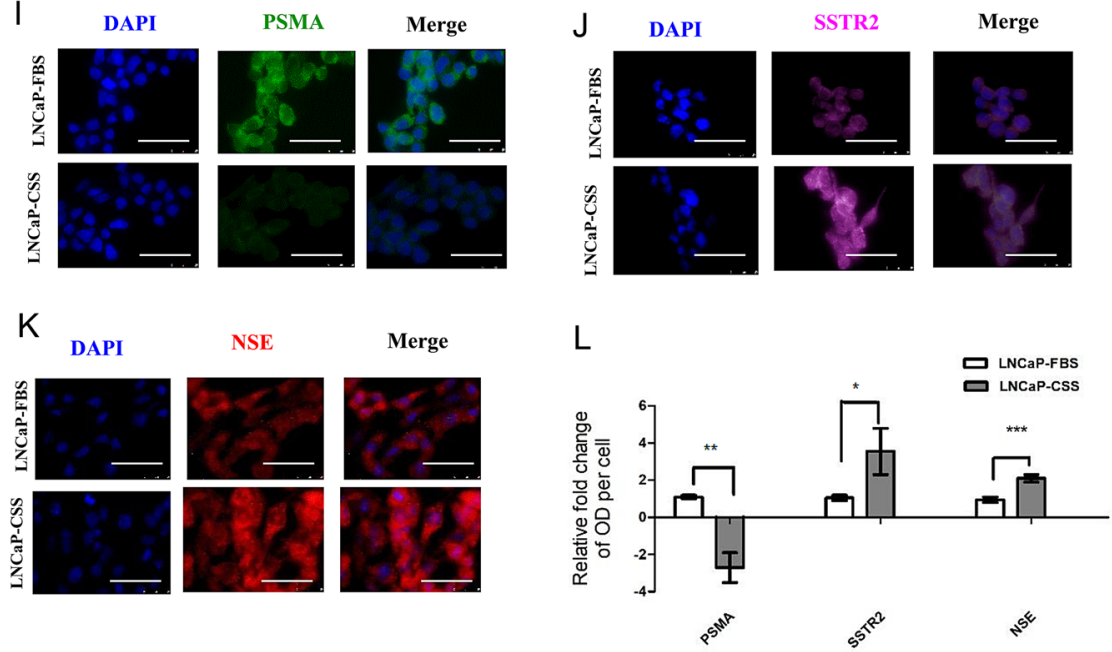

Figure 5

Analysis of PSMA and SSTR2 in a NEPC induced cell line. (A and B) Western blot analyses of protein level of PSMA, SSTR2, AR, NSE and p53 in 3 different prostate cancer cell line models. (A) Immunoblotting (B) diagram showing the relative density of protein levels. (C) Representative photos of control (left) and CSS-treated (right) LNCaP cells stained with Hoechst. Scale bar: $50 \mu \mathrm{m}$. (D, E and F) Neurites were studied under an inverted microscope: (D) \% of cells with neurites counted over 3 fields of view over 3 separate experiments. (E) Neurites were measured using ImageJ software and longest neurite calculated. (F) Average neurite. (G, H, I, J, K and L) LNCaP cells are treated with either FBS or CSS as indicated and level of PSMA, SSTR2, AR, NSE and p53 were detected by $(\mathrm{G}$ and $\mathrm{H})$ immunoblotting and $(\mathrm{I}, \mathrm{J}$ and $\mathrm{K})$ immunocytochemistry. $(\mathrm{L})$ Data are quantified using Image software. Stat: Error bars reflect S.E.M. between three separate experiments. The data were analyzed by either Student's $t$-test or one-way ANOVA followed by a Tukey's multiple comparison tests; $* * P<0.01$ and $* * \star p<0.001$.

\section{Development of ENZ resistance following a p53-dependent suppression of PSMA}

Treatment of LNCaP cell line with ENZ $(10 \mu \mathrm{M})$ resulted in an increase in PSMA protein levels in medium containing either FBS or CSS (Fig. 6A and B). AR levels show no significant alteration under these conditions. Treatment with ENZ in medium supplemented with CSS had an increase in levels of NSE. This experiment, and previously reported data, supports that short-term exposure of LNCaP cell lines to either ENZ treatment or serum removal cannot be a viable approach to make a clinically relevant PSMA-suppressed in vitro model of AdPC with NE features. Recently, it had been reported that p53 knockdown could lead to suppression of luminal markers and overexpression of basal and NE markers (Li et al. 2017, Mu et al. 2017). Figure 6C and D shows p53 knockdown of LNCaP cells in a medium supplemented in 
A
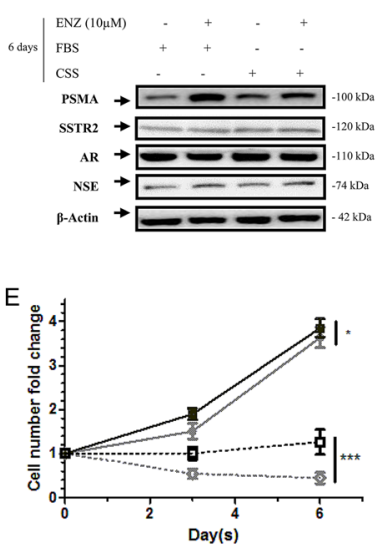

DMSO $[-$ LNCaP-High PSMA

- LNGaP-Low PSMA

ENZ F. LNCaP-High PSMA
B

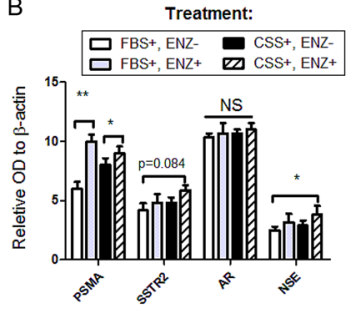

F

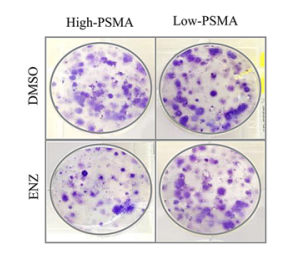

C

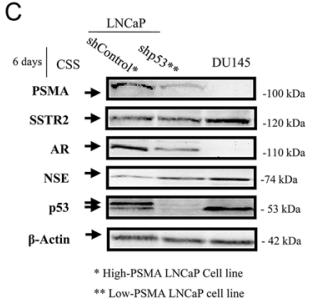

G

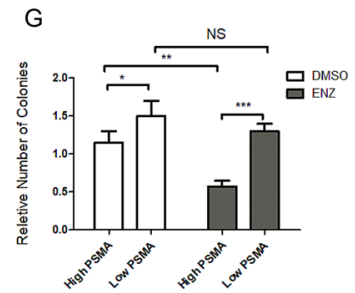

D

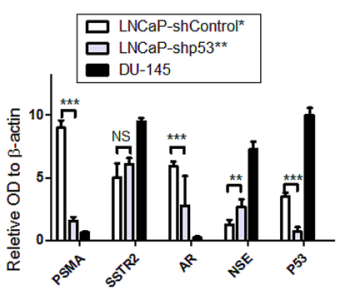

$\mathrm{H}$

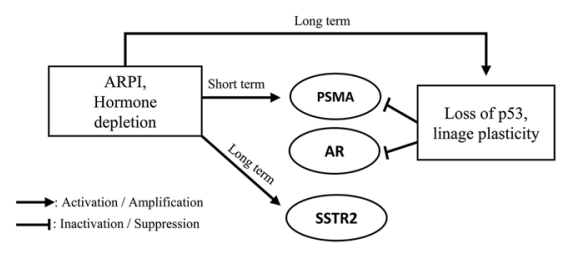

\section{Figure 6}

Analysis of treatment response to ENZ following a p53-dependent suppression of PSMA. (A and B) Western blot analyses of protein level of PSMA, SSTR2, AR and NSE in LNCaP cell line treated with vehicle control (DMSO) or ENZ (10 $\mu \mathrm{M})$ supplemented with either FBS or CSS for 6 days (A) representative immunoblot (B) the relative density of protein levels. (C and D) Western blot analyses of protein level of PSMA, SSTR2, AR, NSE and p53 in LNCaP cell line transduced with annotated shRNA supplemented with CSS for 6 days. (C) Representative immunoblot (D) the relative density of protein levels. (E) Growth curve of LNCaP cell lines with different levels of PSMA following treatment with vehicle control (DMSO) or ENZ (10 $\mu \mathrm{M})$ in supplemented with CSS. ( $F$ and G) The colony-forming ability of high-PSMA and low-PSMA seeded in 10\% CSS for 1 week and treated with either ENZ (10 $\mu$ M) or DMSO for one more week. (F) Representative wells (G) quantification of the number of the colonies using CellProfiler software. (H) Schematic of the impact of ARPI, hormonal deletion and loss of p53 on PSMA, AR and SSTR2 based on the obtained data in Figs 5 and 6. Error bars reflect S.E.M. between three separate experiments. The data were analyzed by either Student's $t$-test or one-way ANOVA followed by a Tukey's multiple comparison tests; **P $P<0.01$ and $* \star \star P<0.001$. A full-colour version of this figure is available at https://doi.org/10.1530/ERC-18-0226.

CSS for 6 days causes suppression AR and PSMA. DU145 as an AR- and PSMA-negative cell line has a significant higher level of NSE $(P<0.01)$. Due to overexpression of NSE and suppression of PSMA, we used the p53-knockdown cell line as a model of low-PSMA cancer with NE features for the next steps of our experiments. The LNCaP cell line transduced with shControl is also referred to as high-PSMA cell line. Figure 6E shows that LNCaP cells with low level of PSMA have slightly higher proliferation during 6-day treatment with the control vehicle (DMSO), but it was not statistically significant $(P=0.65)$. During ENZ $(10 \mu \mathrm{M})$ treatment, low-PSMA cells demonstrate a higher growth rate $(P=0.0048)$ and on the sixth day of cell counting low-PSMA cell numbers were 2.8 times higher than highPSMA cells. It can be inferred that low-PSMA cells are less sensitive to ENZ $(10 \mu \mathrm{M})$ than high-PSMA cells over this 6-day experiment. In addition, we tested sensitivity of LNCaP cells with different levels of PSMA using colony formation assay (Fig. 6F) and find that low-PSMA cells have more colonies formed under ENZ $(10 \mu \mathrm{M})$ treatment than treatment of cells with high PSMA. Therefore, low-PSMA cells were less sensitive to ENZ treatment, supporting the hypothesis that suppression of PSMA, as found in NEPC, correlates with more aggressive disease.

(c) 2019 Society for Endocrinology Published by Bioscientifica Ltd. Printed in Great Britain
Figure $6 \mathrm{H}$ shows a schematic of the associations in changing gene expression found using in vitro models. Hormone depletion, which is modeled by serum deprivation, suppresses PSMA, AR and p53 levels and elevates levels of SSTR2. ARPI which is modeled by treating cells with ENZ showed no alteration of AR or SSTR2 but increased levels of PSMA. A p53 knockdown in vitro model supports that suppression of PSMA and AR are associated with p53-dependent cellular plasticity and that this is independent of SSTR2 levels.

\section{NEPC has a distinct FOLH1-suppressed signature in PDX models}

Following pathological investigation, patient tumors were stratified into either AdPC or NEPC phenotypes put into PDX mouse models and studied before or after castration (schema Fig. 7A, B and C). Significant suppression of FOLH1 was observed in the LTL-331R-G7 and LTL-352 mice, which were found to progress to NEPC, as compared to those mice lacking NE marker expression $(P<0.05)$ (Fig. 7D). There was a wide spectrum of FOLH1 expression in different AdPC models. In the LTL-313-B model, after castration of mice, which can mimic treatment-induced 
A
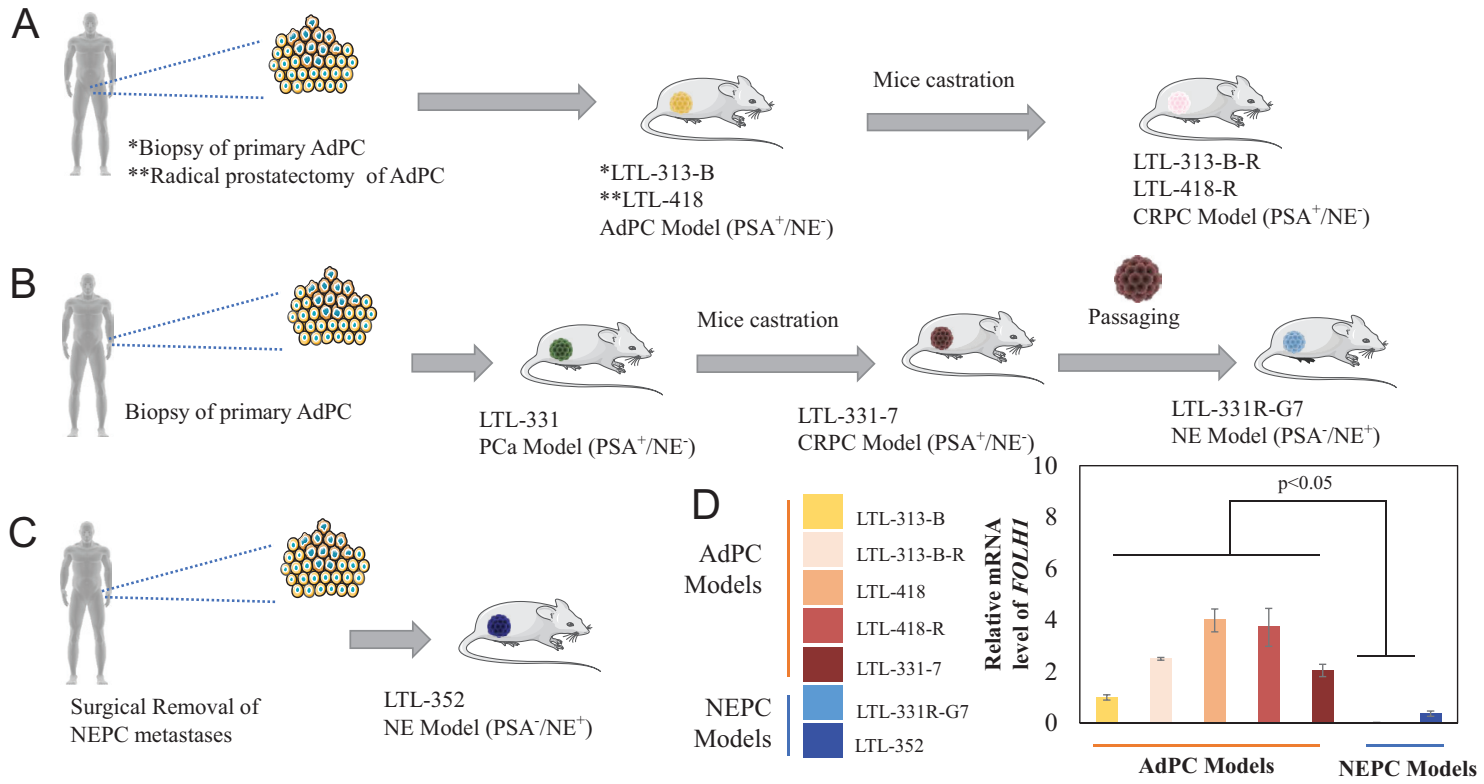

Figure 7

Establishment and analysis of AdPC and NEPC PDX mouse models. (A, B, C and D) Schematic of the established PDX mice models of AdPC and NEPC (adapted, with permission, from Lin et al. (2014)). (D) The levels of FOLH1 in different PDX models. One-way ANOVA followed by a Newman-Keuls multiple comparison test was used $(n=3)$. Some elements of this figure were produced using Servier Medical Art image bank (www.servier.com) under the terms of a Creative Commons Attribution 3.0 Unported licence. A full-colour version of this figure is available at https://doi.org/10.1530/ERC-18-0226.

relapse in the form of CRPC, we observed a 2.4-fold elevation in FOLH1. However, in the LTL-418 model, there was no significant alteration in FOLH1 expression. For simulation of treatment-induced NEPC, after inducing NE to LTL-331-7 model as a CRPC model, a 2.1 times suppression of FOLH1 was observed. Similarly, the PDX model obtained by direct engraftment of tissue from a NEPC metastases sample had significantly lower FOLH1 expression in comparison with all AdPC models.

The transcription of FOLH1 and SSTR2 in 18 PDX models including three NEPCs were investigated (Fig. 8A and B). Among these PDX models, LTL331 and LTL331R are the first-in-field PDX model of AdPC-to-NEPC transdifferentiation. Figure 8C illustrates upon LTL331 model castration, the primary AdPC initially regresses but relapses within 24-32 months as typical CRPC and the whole transdifferentiation process is predictive of disease progression and is fully recapitulated in the donor patient (Akamatsu et al. 2015, Ci et al. 2018). Figure 8B shows a five-fold suppression of FOLH1 gene expression in NEPC models subsequent with a two fold increase in the expression of SSTR2. Figure 8D shows of the expression levels of FOLH1 and SSTR2 through the progression to NEPC. In the AdPC model, FOLH1 is found at a maximum level and SSTR2 at a minimum level. Following castration up to 8 weeks there is low fluctuations in the transcription of both of the genes. However, on 8 th week, a sharp decline in FOLH1 and a profound elevation of SSTR2 starts. The terminally differentiated NEPC model has a minimal transcriptional level of FOLH1 and maximal transcription of SSTR2.

\section{PSMA levels fail to adequately predict NE transdifferentiation of high-grade AdPC}

Similar to its RNA, PSMA protein level could have a variable series of staining levels of low grade (Supplementary Fig. 5A and D), medium grade (Supplementary Fig. 5B and E) and high grade (Supplementary Fig. 5C and F) AdPC. These staining patterns imply a series of possible progression paths for AdPC progression and eventual NEPC. On Fig. 8E, we proposed five possible models for alteration of PSMA during progression of AdPC. Model 1 is the classical model of PSMA expression in which the level of PSMA correlates positively with the stage of AdPC, our data support that this model may not adequately represent all stages of disease. Model 5 is a representation of a de novo occurrence of NEPC, which shows poor-onset PSMA staining. However, models 2 and 3 are relevant as AdPC progression pathways that lead to NE transdifferentiation and subsequent loss of PSMA. In addition, model 4 is representation of a constant poor PSMA staining, which against its low abundance is still capable of detecting metastatic sites. The HPA dataset PSMA antibody staining 
A

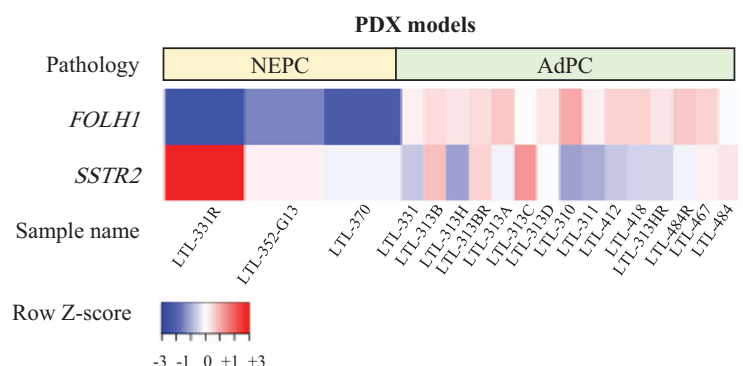

B

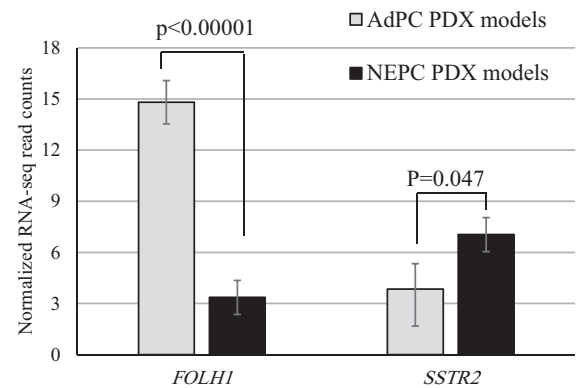

C
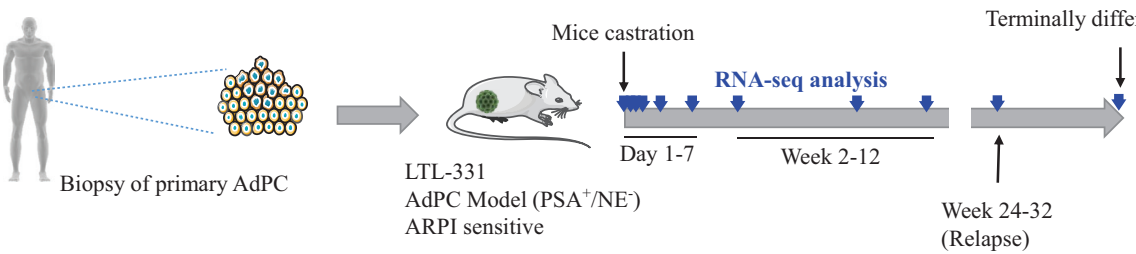

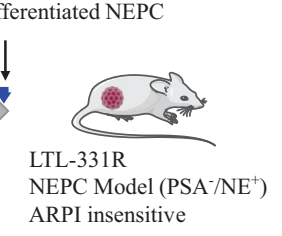

D

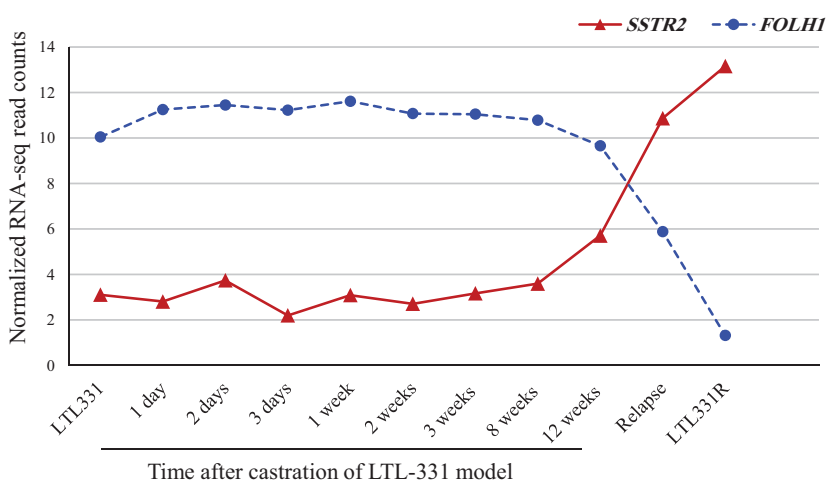

E

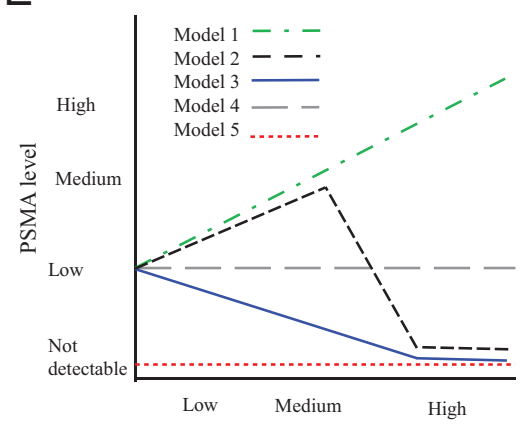

Prostate Adenocarcinoma grade

\section{Figure 8}

NEPC represents a distinctive FOLH1-supressed signature in a series of PDX model. (A and B) Transcriptomic profiles from the PDX models (15 adenocarcinomas vs 3 NEPCS), (A) heatmap showing the clustering among all PDX samples (B) the average level of FOLH1 and SSTR2 suggests a unique downregulation of FOLH1 in NEPC PDX tumors while SSTR2 levels are slightly increased. The data were analyzed by Student's $t$-test. (C) Schematic of development of LTL-331R as terminally differentiated NEPC PDX model following castration of hormone-sensitive LTL-331 PDX model. The time points at which tumors were collected along progression to NEPC are illustrated by blue color arrows. Some elements of this figure were produced using Servier Medical Art image bank (www.servier.com) under the terms of a Creative Commons Attribution 3.0 Unported licence. (D) Transcription of FOLH1 and SSTR2 during NE transdifferentiation in the LTL331 system highlighting the suppression of FOLH1 and amplification of SSTR2 during development of NEPC as a result of hormone depletion. (E) Possible models of alteration of PSMA level during progression of AdPC. Schema shows possible kinetic changes in PSMA level ( $Y$ axis) during progression from low-, medium- and high-grade AdPC. A full-colour version of this figure is available at https://doi.org/10.1530/ ERC-18-0226.

patterns for AdPC progression led us to conclude that model 2 is the most likely progression pattern for the treatment-induced NEPC.

\section{Discussion}

The development of NE phenotype following hormone depletion in LNCaP cell line is a well-studied observation (Shen et al. 1997, Yuan et al. 2006). Liu et al. (2012) reported that hormone depletion suppresses AR and PSMA levels in LNCaP cells; however, they did not evaluate the expression of NE makers. Suppression of AR and overexpression of
NE makers in LNCaP following p53 knockdown in LNCaP cell has also been described recently (Li et al. 2017, Mu et al. 2017). In this manuscript, we report the suppression of PSMA and overexpression of NSE and SSTR2 in LNCaP cell line following hormone depletion and loss of p53.

Mannweiler et al. reported that a direct correlation between histological parameters and PSMA could not be established; specifically, in AdPC patients with distant metastasis (Mannweiler et al. 2009). In this study, we report the possibility of PSMA suppression in high grades of CRPC. We further show that expression of the PSMA gene, FOLH1, inversely correlates with markers of NE 
differentiation. These data support previous clinical reports showing a suppression of PSMA-targeted nuclear scans for NEPC patients (Chakraborty et al. 2015, Tosoian et al. 2017, Usmani et al.2017). This mounts the possibility that portions of AdPC patients undergoing ARPI will transdifferentiate to NEPC and will subsequently fail to adequately uptake PSMA-targeted radiopharmaceuticals.

Clinical reports show the possibility of a false-positive uptake of PSMA-targeted radioligand after ARPI (Hope et al. 2017) and a false-negative uptake of this radioligand in NEPC (Chakraborty et al. 2015, Tosoian et al. 2017, Usmani et al. 2017). We previously reviewed the available preclinical evidence justifying molecular backgrounds of a false-positive uptake of PSMA-targeted radioligand after ARPI (Bakht et al. 2017). However, there is a lack of preclinical evidence for the reported false-negative uptake of PSMA-targeted radioligand. The observed significant suppression of FOLH1 expression in PDX models shown in this work are the first preclinical evidence validating false-negative uptake of PSMA-targeted radioligand in NEPC. The suggested second model of alteration of PSMA on Fig. 8E best describes the progression of AdPC patients undergoing ARPI who end up with NEPC.

There is literature to support that SSTR-targeted radioligands such as ${ }^{68} \mathrm{Ga}$-DOTATOC or ${ }^{68} \mathrm{Ga}$-DOTATATE could be valuable alternatives for PSMA-negative and non-hormone-naive patients. Usmani et al. compared ${ }^{68} \mathrm{Ga}$-PSMA vs ${ }^{68} \mathrm{Ga}$-DOTA NOC PET-CT in a 62 -year-old NEPC patient and reported that the NEPC tumor and its metastatic lesions have avid uptake of ${ }^{68} \mathrm{Ga}$-DOTA NOC while the PSMA uptake was faint and inconclusive (Usmani et al. 2017). Additionally, in a study on 12 patients with CRPC, ${ }^{68} \mathrm{Ga}$-DOTATATE PET/CT is suggested for NEPC imaging and early detection of metastatic lymph node and blastic or lytic bone lesions (Gofrit et al. 2017, Sheikhbahaei et al. 2017). In this study, we report an overexpression of SSTR2 in NEPC that could potentially lead to overexpression of SSTR2 at the protein level and positivity in the uptake of SSTR radioligand. Interestingly, Morichetti et al. reported a strong specific staining for SSTR2 in 20 cases of NEPC (Morichetti et al. 2010). We speculate that SSTR radioligand might be a feasible tool to delineate PSMA-negative NEPC from the normal tissue while PSMAnegative NEPC is not detectable by PSA measurements or by PSMA radiotracers. Future work to explore the role of SSTR2-targeted radionuclide therapy for the detection and/or management of NEPC is of high priority.

Two possible scenarios for a patient with a suppressed PSMA radioligand uptake after ARPI are illustrated on Supplementary Fig. 6. In an ideal condition, the PSMA radioligand should be able to delineate AdPC tumor and all metastatic lesions (Supplementary Fig. 6A); consequently, after therapy faint or a no PSMA radioligand uptake should be observed since ARPI suppresses the malignancy (Supplementary Fig. 6B). However, as a second possible scenario, we might face a high-grade NEPC with suppressed PSMA expression level (Supplementary Fig. 6C). In this case, SSTR2 radioligand might be able to delineate NEPC tumor and its metastatic lesions.

\section{Conclusion}

The use of potent antiandrogens and lineage plasticity may contribute to the increasing prevalence of NEPC, an aggressive and hormone-resistant form of AdPC. While PSMA targeting is a promising approach for the nuclear imaging and therapy of many forms of aggressive AdPC, our data based on transcriptome analysis of tumor samples, cell line models and PDX mice models supports that PSMA (FOLH1 gene) levels are not consistent with all forms of high-grade CRPC. Our in vitro data showed a significant suppression of PSMA as a result of hormone depletion, loss of p53 and lineage plasticity. A PSMAsuppressed NEPC cell line model showed higher colony formation and resistance to ARPI by ENZ. Our data further show that induced NE transdifferentiation correlates with an elevated level of the protein SSTR2. SSTR2 levels appear to elevate due to hormone depletion but are not affected by altered cellular plasticity. PDX data support these conclusions, showing an inverse correlation between the expression of the PSMA gene and SSTR2 gene. Specifically we find declining PSMA and increasing SSTR2 gene expression occurring during the development of CRPC and becoming more pronounced in terminally differentiated NEPC. Collectively, this study cautions on the reliability of using PMSA levels as a diagnostic target for molecular imaging in advanced treatment-induced NEPC. Our work supports the recommendation that SSTR2-targeted imaging approaches may permit more accurate monitoring of PSMA-suppressed patients due to SSTR2-positivity of NEPC tumors.

\section{Supplementary data}

This is linked to the online version of the paper at https://doi.org/10.1530/ ERC-18-0226.

\section{Declaration of interest}

The authors declare that there is no conflict of interest that could be perceived as prejudicing the impartiality of the research reported. 


\section{Funding}

This research was supported by a grant of the Korea Health Technology R\&D Project through the Korea Health Industry Development Institute $(\mathrm{KHIDI})$, funded by the Ministry of Health \& Welfare, Republic of Korea (grant number: HI14C1072). The financial support of Prostate Cancer Fight Foundation (Motorcycle Ride for Dad Charity, ON, Canada) and Ontario Trillium Scholarship program (Government of Ontario, ON, Canada) is greatly appreciated.

\section{Author contribution statement}

The conception and design of the study was by M K B, G J C, A D L, K F S, $K W K, Y W, X D$ and $L A P . M K B, I D, Y L$ and $Y W$ did the experiments and obtained the data. M KB, I D, Y L, A H and A D L analyzed the data. M KB, ID, R M F, S W O, H Y and L A P drafted the manuscript. M K B, R F, H Y, K F S, CW J, G J C, CK, M D, KW K, A D L, XD and L A P revised the manuscript.

\section{References}

Akamatsu S, Wyatt AW, Lin D, Lysakowski S, Zhang F, Kim S, Tse C, Wang K, Mo F \& Haegert A 2015 The placental gene PEG10 promotes progression of neuroendocrine prostate cancer. Cell Reports 12 922-936. (https://doi.org/10.1016/j.celrep.2015.07.012)

Al Sorkhy M, Ferraiuolo R-M, Jalili E, Malysa A, Fratiloiu AR, Sloane BF \& Porter LA 2012 The cyclin-like protein Spy1/RINGO promotes mammary transformation and is elevated in human breast cancer. BMC Cancer 12 45. (https://doi.org/10.1186/1471-2407-12-45)

Bakht MK, Oh SW, Youn H, Cheon GJ, Kwak C \& Kang KW 2017 Influence of androgen deprivation therapy on the uptake of PSMAtargeted agents: emerging opportunities and challenges. Nuclear Medicine and Molecular Imaging 51 202-211. (https://doi.org/10.1007/ s13139-016-0439-4)

Beltran H, Prandi D, Mosquera JM, Benelli M, Puca L, Cyrta J, Marotz C, Giannopoulou E, Chakravarthi BV \& Varambally S 2016 Divergent clonal evolution of castration-resistant neuroendocrine prostate cancer. Nature Medicine 22 298-305. (https://doi.org/10.1038/ nm.4045)

Boegemann M, Schrader AJ \& Rahbar K 2017 [177Lu-PSMA therapy: current evidence for use in the treatment of patients with metastatic prostate cancer]. Urologe Ausgabe A 56 1440-1444. (https://doi. org/10.1007/s00120-017-0510-5)

Chakraborty PS, Tripathi M, Agarwal KK, Kumar R, Vijay MK \& Bal C 2015 Metastatic poorly differentiated prostatic carcinoma with neuroendocrine differentiation: negative on ${ }^{68} \mathrm{Ga}$-PSMA PET/CT. Clinical Nuclear Medicine 40 e163-e166. (https://doi.org/10.1097/ RLU.0000000000000594)

Chandrashekar DS, Bashel B, Balasubramanya SAH, Creighton CJ, Ponce-Rodriguez I, Chakravarthi BVSK \& Varambally S 2017 UALCAN: a portal for facilitating tumor subgroup gene expression and survival analyses. Neoplasia 19 649-658. (https://doi. org/10.1016/j.neo.2017.05.002)

Chappell WH, Lehmann BD, Terrian DM, Abrams SL, Steelman LS \& McCubrey JA 2012 p53 expression controls prostate cancer sensitivity to chemotherapy and the MDM2 inhibitor Nutlin-3. Cell Cycle 11 4579-4588. (https://doi.org/10.4161/cc.22852)

Chen R, Dong X \& Gleave M 2018 Molecular model for neuroendocrine prostate cancer progression. BJU International 122 560-570. (https:// doi.org/10.1111/bju.14207)

Ci X, Hao J, Dong X, Choi SY, Xue H, Wu R, Qu S, Gout PW, Zhang F, Haegert AM, et al. 2018 Heterochromatin protein 1alpha mediates development and aggressiveness of neuroendocrine prostate cancer. Cancer Research 78 2691-2704. (https://doi.org/10.1158/0008-5472. CAN-17-3677) da Silva EF, Botsford S \& Porter LA 2016 Derivation of a novel G2 reporter system. Cytotechnology 68 19-24. (https://doi.org/10.1007/ s10616-014-9757-5)

Davies AH, Beltran H \& Zoubeidi A 2018 Cellular plasticity and the neuroendocrine phenotype in prostate cancer. Nature Reviews Urology 15 271-286. (https://doi.org/10.1038/nrurol.2018.22)

Ding Y, Li Y, Lu L, Zhang R, Zeng L, Wang L \& Zhang X 2015 Inhibition of nischarin expression promotes neurite outgrowth through regulation of PAK activity. PLOS ONE 10 e0144948. (https://doi. org/10.1371/journal.pone.0144948)

Dunning MJ, Vowler SL, Lalonde E, Ross-Adams H, Boutros P, Mills IG, Lynch AG \& Lamb AD 2017 Mining human prostate cancer datasets: the "camcAPP" shiny app. EBioMedicine 17 5-6. (https://doi. org/10.1016/j.ebiom.2017.02.022)

Gao J, Aksoy BA, Dogrusoz U, Dresdner G, Gross B, Sumer SO, Sun Y, Jacobsen A, Sinha R \& Larsson E 2013 Integrative analysis of complex cancer genomics and clinical profiles using the cBioPortal. Science Signaling 6 pl1. (https://doi.org/10.1126/scisignal.2004088)

Gofrit ON, Frank S, Meirovitz A, Nechushtan H \& Orevi M 2017 PET/CT with ${ }^{68} \mathrm{Ga}$-DOTA-TATE for diagnosis of neuroendocrine: differentiation in patients with castrate-resistant prostate cancer. Clinical Nuclear Medicine 42 1-6. (https://doi.org/10.1097/ RLU.0000000000001424)

Grasso CS, Wu Y-M, Robinson DR, Cao X, Dhanasekaran SM, Khan AP, Quist MJ, Jing X, Lonigro RJ, Brenner JC, et al. 2012 The mutational landscape of lethal castration-resistant prostate cancer. Nature $\mathbf{4 8 7}$ 239-243. (https://doi.org/10.1038/nature11125)

Hope TA, Truillet C, Ehman EC, Afshar-Oromieh A, Aggarwal R, Ryan CJ, Carroll PR, Small EJ \& Evans MJ 2017 68Ga-PSMA-11 PET imaging of response to androgen receptor inhibition: first human experience. Journal of Nuclear Medicine 58 81-84. (https://doi. org/10.2967/jnumed.116.181800)

Hothorn T, Hornik K \& Zeileis A 2006 Unbiased recursive partitioning: a conditional inference framework. Journal of Computational and Graphical Statistics 15 651-674. (https://doi.org/10.1198/1061860 06X133933)

Jadvar H \& Ballas LK 2018 PSMA PET: transformational change in prostate cancer management? Journal of Nuclear Medicine $\mathbf{5 9}$ 228-229. (https://doi.org/10.2967/jnumed.117.203604)

Kopka K, Benešová M, Bařinka C, Haberkorn U \& Babich J 2017 Gluureido-based inhibitors of prostate-specific membrane antigen: lessons learned during the development of a novel class of lowmolecular-weight theranostic radiotracers. Journal of Nuclear Medicine 58 17S-26S. (https://doi.org/10.2967/jnumed.116.186775)

Li W, Cohen A, Sun Y, Squires J, Braas D, Graeber TG, Du L, Li G, Li Z, $\mathrm{Xu}$ X, et al. 2016 The role of CD44 in glucose metabolism in prostatic small cell neuroendocrine carcinoma. Molecular Cancer Research 14 344-353. (https://doi.org/10.1158/1541-7786.MCR-150466)

Li Y, Donmez N, Sahinalp C, Xie N, Wang Y, Xue H, Mo F, Beltran H, Gleave M, Wang Y, et al. 2017 SRRM4 drives neuroendocrine transdifferentiation of prostate adenocarcinoma under androgen receptor pathway inhibition. European Urology 71 68-78. (https://doi. org/10.1016/j.eururo.2016.04.028)

Lin D, Wyatt AW, Xue H, Wang Y, Dong X, Haegert A, Wu R, Brahmbhatt S, Mo F \& Jong L 2014 High fidelity patient-derived xenografts for accelerating prostate cancer discovery and drug development. Cancer Research 74 1272-1283. (https://doi. org/10.1158/0008-5472.CAN-13-2921-T)

Liu T, Wu LY, Fulton MD, Johnson JM \& Berkman CE 2012 Prolonged androgen deprivation leads to downregulation of androgen receptor and prostate-specific membrane antigen in prostate cancer cells. International Journal of Oncology 41 2087-2092. (https://doi. org/10.3892/ijo.2012.1649)

Mannweiler S, Amersdorfer P, Trajanoski S, Terrett JA, King D \& Mehes G 2009 Heterogeneity of prostate-specific membrane antigen 
(PSMA) expression in prostate carcinoma with distant metastasis. Pathology Oncology Research 15 167-172. (https://doi.org/10.1007/ s12253-008-9104-2)

Morichetti D, Mazzucchelli R, Santinelli A, Stramazzotti D, LopezBeltran A, Scarpelli M, Bono A, Cheng L \& Montironi R 2010 Immunohistochemical expression and localization of somatostatin receptor subtypes in prostate cancer with neuroendocrine differentiation. International Journal of Immunopathology and Pharmacology 23 511-522. (https://doi. org/10.1177/039463201002300213)

Mu P, Zhang Z, Benelli M, Karthaus WR, Hoover E, Chen CC, Wongvipat J, Ku SY, Gao D, Cao Z, et al. 2017 SOX2 promotes lineage plasticity and antiandrogen resistance in TP53- and RB1deficient prostate cancer. Science 355 84-88. (https://doi.org/10.1126/ science.aah4307)

Nabavi N, Saidy NRN, Venalainen E, Haegert A, Parolia A, Xue H, Wang Y, Wu R, Dong X \& Collins C 2017 miR-100-5p inhibition induces apoptosis in dormant prostate cancer cells and prevents the emergence of castration-resistant prostate cancer. Scientific Reports $\mathbf{7}$ 4079. (https://doi.org/10.1038/s41598-017-03731-8)

Parida GK, Tripathy S, Datta Gupta S, Singhal A, Kumar R, Bal C \& Shamim SA 2018 Adenocarcinoma prostate with neuroendocrine differentiation: potential utility of 18F-FDG PET/CT and 68Ga-DOTANOC PET/CT over 68Ga-PSMA PET/CT. Clinical Nuclear Medicine 43 248-249. (https://doi.org/10.1097/RLU.0000000000002013)

Parimi V, Goyal R, Poropatich K \& Yang XJ 2014 Neuroendocrine differentiation of prostate cancer: a review. American Journal of Clinical and Experimental Urology 2 273-285.

Poeppel TD, Binse I, Petersenn S, Lahner H, Schott M, Antoch G, Brandau W, Bockisch A \& Boy C 2011 68Ga-DOTATOC versus 68Ga-DOTATATE PET/CT in functional imaging of neuroendocrine tumors. Journal of Nuclear Medicine 52 1864-1870. (https://doi. org/10.2967/jnumed.111.091165)

Rai BP, Baum R, Patel A, Hughes R, Alonzi R, Lane T, Adshead J \& Vasdev N 2016 The role PET with 68 gallium (Ga)-labelled prostatespecific membrane antigen (PSMA) in the Management of Patient with organ confined and locally advanced prostate cancer prior to radical treatment and after radical prostatectomy. Urology 95 11-15. (https://doi.org/10.1016/j.urology.2015.12.048)

Ross-Adams H, Lamb AD, Dunning MJ, Halim S, Lindberg J, Massie CM, Egevad LA, Russell R, Ramos-Montoya A, Vowler SL, et al. 2015 Integration of copy number and transcriptomics provides risk stratification in prostate cancer: a discovery and validation cohort study. EBioMedicine 2 1133-1144. (https://doi.org/10.1016/j. ebiom.2015.07.017)

Rowe SP, Gorin MA \& Pomper MG 2017 Imaging of prostate-specific membrane antigen using [18F] DCFPyL. PET Clinics 12 289-296. (https://doi.org/10.1016/j.cpet.2017.02.006)

Sheikhbahaei S, Afshar-Oromieh A, Eiber M, Solnes LB, Javadi MS, Ross AE, Pienta KJ, Allaf ME, Haberkorn U, Pomper MG, et al. 2017
Pearls and pitfalls in clinical interpretation of prostate-specific membrane antigen (PSMA)-targeted PET imaging. European Journal of Nuclear Medicine and Molecular Imaging 44 2117-2136. (https://doi. org/10.1007/s00259-017-3780-7)

Shen R, Dorai T, Szaboles M, Katz AE, Olsson CA \& Buttyan R 1997 Transdifferentiation of cultured human prostate cancer cells to a neuroendocrine cell phenotype in a hormone-depleted medium. Urologic Oncology: Seminars and Original Investigations 3 67-75. (https://doi.org/10.1016/S1078-1439(97)00039-2)

Taylor BS, Schultz N, Hieronymus H, Gopalan A, Xiao Y, Carver BS, Arora VK, Kaushik P, Cerami E, Reva B, et al. 2010 Integrative genomic profiling of human prostate cancer. Cancer Cell 18 11-22. (https://doi.org/10.1016/j.ccr.2010.05.026)

Thul PJ, Åkesson L, Wiking M, Mahdessian D, Geladaki A, Ait Blal H, Alm T, Asplund A, Björk L, Breckels LM, et al. 2017 A subcellular map of the human proteome. Science $\mathbf{3 5 6}$ eaal3321. (https://doi. org/10.1126/science.aal3321)

Tosoian JJ, Gorin MA, Rowe SP, Andreas D, Szabo Z, Pienta KJ, Pomper MG, Lotan TL \& Ross AE 2017 Correlation of PSMA-targeted (18)F-DCFPyL PET/CT findings with immunohistochemical and genomic data in a patient with metastatic neuroendocrine prostate cancer. Clinical Genitourinary Cancer 15 e65-e68. (https://doi. org/10.1016/j.clgc.2016.09.002)

Uhlen M, Zhang C, Lee S, Sjöstedt E, Fagerberg L, Bidkhori G, Benfeitas R, Arif M, Liu Z, Edfors F, et al. 2017 A pathology atlas of the human cancer transcriptome. Science $\mathbf{3 5 7}$ eaan2507. (https:// doi.org/10.1126/science.aan2507)

Unni E, Sun S, Nan B, McPhaul MJ, Cheskis B, Mancini MA \& Marcelli M 2004 Changes in androgen receptor nongenotropic signaling correlate with transition of LNCaP cells to androgen independence. Cancer Research 64 7156-7168. (https://doi. org/10.1158/0008-5472.CAN-04-1121)

Usmani S, Ahmed N, Marafi F, Rasheed R, Amanguno HG \& Al Kandari F 2017 Molecular imaging in neuroendocrine differentiation of prostate cancer: ${ }^{68} \mathrm{Ga}$-PSMA versus ${ }^{68} \mathrm{Ga}$-DOTA NOC PET-CT. Clinical Nuclear Medicine 42 410-413. (https://doi.org/10.1097/ RLU.0000000000001618)

Vlachostergios PJ \& Papandreou CN 2015 Targeting neuroendocrine prostate cancer: molecular and clinical perspectives. Frontiers in Oncology 5 6. (https://doi.org/10.3389/fonc.2015.00006)

Yuan TC, Veeramani S, Lin FF, Kondrikou D, Zelivianski S, Igawa T, Karan D, Batra SK \& Lin MF 2006 Androgen deprivation induces human prostate epithelial neuroendocrine differentiation of androgen-sensitive LNCaP cells. Endocrine-Related Cancer 13 151-167. (https://doi.org/10.1677/erc.1.01043)

Zelivianski S, Verni M, Moore C, Kondrikov D, Taylor R \& Lin M-F 2001 Multipathways for transdifferentiation of human prostate cancer cells into neuroendocrine-like phenotype. Biochimica et Biophysica Acta (BBA) - Molecular Cell Research 1539 28-43. (https://doi. org/10.1016/S0167-4889(01)00087-8)

Received in final form 24 September 2018

Accepted 11 October 2018

Accepted Preprint published online 11 October 2018 https://erc.bioscientifica.com

https://doi.org/10.1530/ERC-18-0226 (c) 2019 Society for Endocrinology Published by Bioscientifica Ltd. Printed in Great Britain 